\title{
Interfraction and intrafraction performance of the Gamma Knife Extend system for patient positioning and immobilization
}

\author{
Clinical article
}

\author{
David Schlesinger, Ph.D., ${ }^{1,2}$ Zhiyuan Xu, M.D., ${ }^{2}$ Frances Taylor, B.A., R.T.(R)(T), ${ }^{1}$ \\ Chun-Po Yen, M.D., ${ }^{2}$ and Jason Sheehan, M.D., Ph.D. ${ }^{1,2,3}$ \\ Departments of ${ }^{1}$ Radiation Oncology, ${ }^{2}$ Neurological Surgery, and ${ }^{3}$ Neuroscience, University of Virginia \\ Health System, Charlottesville, Virginia
}

\begin{abstract}
Object. The Extend system for the Gamma Knife Perfexion makes possible multifractional Gamma Knife treatments. The Extend system consists of a vacuum-monitored immobilization frame and a positioning measurement system used to determine the location of the patient's head within the frame at the time of simulation imaging and before each treatment fraction. The measurement system consists of a repositioning check tool (RCT), which attaches to the Extend frame, and associated digital measuring gauges. The purpose of this study is to evaluate the performance of the Extend system for patient repositioning before each treatment session (fraction) and patient immobilization between (interfraction) and during (intrafraction) each session in the first 10 patients (36 fractional treatments) treated at the University of Virginia.

Methods. The RCT was used to acquire a set of reference measurements for each patient position at the time of CT simulation. Repositioning measurements were acquired before each fraction, and the patient position was adjusted until the residual radial difference from the reference position measurements was less than $1 \mathrm{~mm}$. After treatment, patient position measurements were acquired, and the difference between those measurements and the ones obtained for patient position before the fraction was calculated as a measure of immobilization capability.

Analysis of patient setup and immobilization performance included calculation of the group mean, standard deviation (SD), and distribution of systematic (components affecting all fractions) and random (per fraction) uncertainty components.

Results. Across all patients and fractions, the mean radial setup difference from the reference measurements was $0.64 \mathrm{~mm}$, with an SD of $0.24 \mathrm{~mm}$. The distribution of systematic uncertainty $(\Sigma)$ was $0.17 \mathrm{~mm}$, and the distribution of random uncertainty $(\sigma)$ was $0.16 \mathrm{~mm}$. The root mean square (RMS) differences for each plate of the RCT were as follows: right $=0.35 \mathrm{~mm}$; left $=0.41 \mathrm{~mm}$; superior $=0.28 \mathrm{~mm}$; and anterior $=0.20 \mathrm{~mm}$.

The mean intrafractional positional difference across all treatments was $0.47 \mathrm{~mm}$, with an SD of $0.30 \mathrm{~mm}$. The distribution of systematic uncertainty was $0.18 \mathrm{~mm}$, and the distribution of random uncertainty was $0.22 \mathrm{~mm}$. The RMS differences for each plate of the RCT were $0.24 \mathrm{~mm}$ for the right plate, $0.22 \mathrm{~mm}$ for the left plate, $0.24 \mathrm{~mm}$ for the superior plate, and $0.34 \mathrm{~mm}$ for the anterior plate. Data from 1 fraction were excluded from the analysis because the vacuum-monitoring interlock detected patient motion, which in turn required repositioning in the middle of the fraction.

Conclusions. The Extend system can be used to reposition and immobilize patients in a radiosurgical setting. However, care should be taken to acquire measurements that can implicitly account for rotations of the patient's head. Further work is required to determine the sensitivity of the vacuum interlock to detect patient motion. (http://thejns.org/doi/abs/10.3171/2012.6.GKS12989)
\end{abstract}

\section{Key Words - Gamma Knife surgery - Extend System • relocatable frame • multifraction radiosurgery • stereotactic radiosurgery • uncertainty analysis}

$\mathrm{T}$ HE primary goal of any radiosurgical system is to deliver extremely conformal, high doses of radiation to a specified target while minimizing the dose

\footnotetext{
Abbreviations used in this paper: CTV = clinical target volume; GKS = Gamma Knife surgery; LED = light-emitting diode; PCU = patient control unit; RCT = Repositioning Check Tool; RMS = root mean square; $\mathrm{SRS}=$ stereotactic radiosurgery.
}

to surrounding anatomical structures. Implicit in this goal is a high level of precision required during setup and immobilization of the patient so the target does not deviate from its intended position (Fig. 1). To this end, intracranial radiosurgical systems have traditionally involved invasive frame systems that serve to immobilize the patient's head during treatment and also establish a coordinate system to facilitate precision delivery of radiation to the target. ${ }^{16}$ 

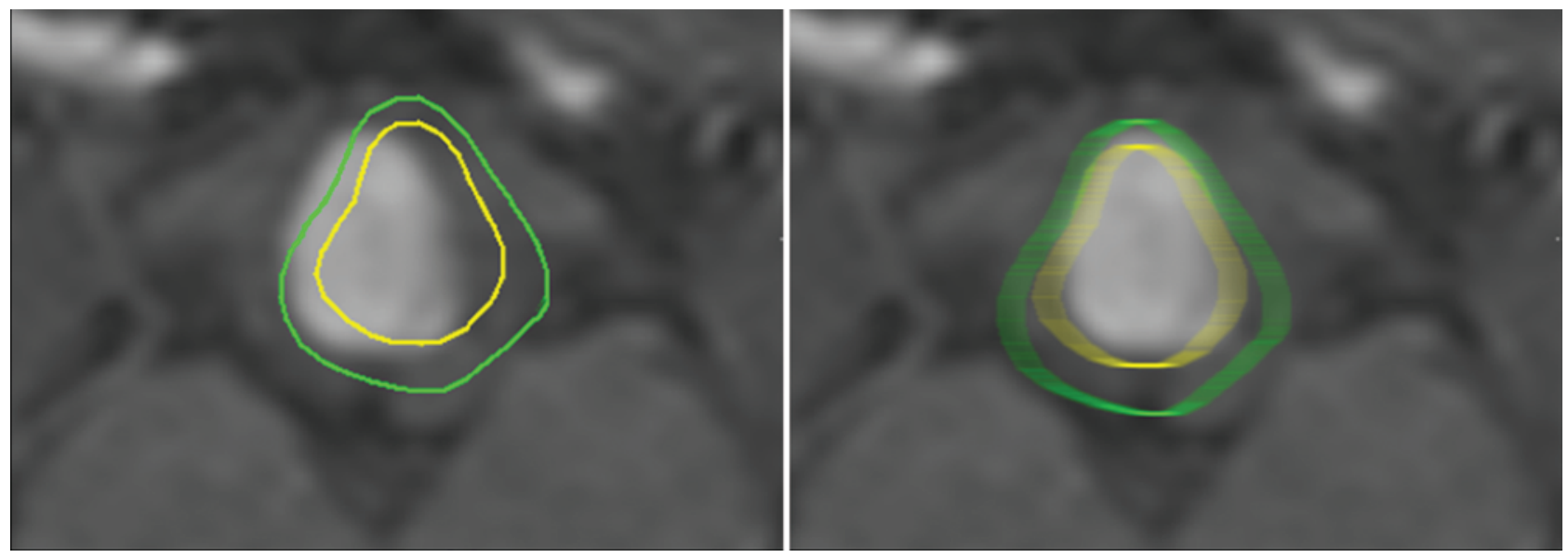

FIG. 1. Images used to show sources of uncertainty in stereotactic radiosurgery that are directly related to the immobilization system. Left: Systematic uncertainty. The target moves away from the planned position in a similar direction over all fractions; the effect is geometrically to "miss" part of the target. Right: Random uncertainty. The target moves randomly in each fraction; the effect is to blur the dose delivered to the target.

Gamma Knife surgery traditionally has been a single-session procedure involving such a rigid frame-based technique. ${ }^{24}$ The recently released Extend system for the Gamma Knife Perfexion (Elekta AB) makes possible multisession GKS procedures, including hypofractionated radiation treatments of $1-5$ dose fractions for individual targets or repeated procedures to treat multiple, anatomically distinct targets. Repeated placement of an invasive frame in these scenarios is impractical. The Extend system removes the need for pin-based frame fixation. ${ }^{15}$ Instead of using a rigid stereotactic frame, the Extend System uses a vacuum-assisted dental fixation device mounted on a carbon-fiber frame to obtain noninvasive immobilization. A vac-lock-type vacuum cushion is placed posterior to the patient's head to help determine a comfortable, reproducible position. A vacuum pump, known as the "patient control unit" or PCU, serves to create suction between the patient's dental impression and the patient's palate. The vacuum level is monitored by the system to alert the operator if the suction level decreases by a threshold amount, which acts as a surrogate for patient motion. The vacuum monitor is interlocked to the treatment unit to pause treatment should motion occur.

Because the Gamma Knife Perfexion does not include onboard image guidance, a method is required to determine the patient's position at the time of treatment relative to the patient's position at the time of treatment planning. Using the Extend system, this is accomplished through the use of linear measurement probes (C150XB Digimatic Indicator, Mitutoyo Corp.) and a measurement template called the "repositioning check tool (RCT)." The RCT mounts on the Extend frame over the patient's head and contains a number of measurement locations on its superior, anterior, left, and right plates, which allow measurements to be taken using the measurement probes.

The flexibility in treatment afforded by the Extend system comes with the tradeoff of a potential loss of accuracy in setup and immobilization. ${ }^{17}$ While data regarding the performance of a beta version of this new system has been published previously, ${ }^{13}$ to our knowledge there is no publication describing the production version of the Extend system in its intended clinical setting. To this end, the object of this study was to quantify the uncertainty for patient setup and patient immobilization when the Extend system is used.

\section{Methods}

Positioning measurements for 10 consecutively enrolled patients (for a total of 36 fractions) treated at the University of Virginia between January 2010 and August 2011 were used to estimate setup and immobilization uncertainties. Table 1 displays the demographics of the patients, including patient sex and age, diagnosis, tumor volume, and beam time at the time of treatment planning.

\section{Extend Setup and Simulation}

The general process for treatment with the Extend system has been described previously. ${ }^{15}$ In summary, preparation for treatment with the Extend system involves the formation of a dental impression of the patient's upper palate, creation of a patient-specific Extend frame setup and head cushion, acquisition of volumetric MR images for treatment planning, acquisition of stereotactic CT scans to compute stereotactic coordinates, coregistration of the MR and CT images, and treatment planning.

At our center, the dental impression is created with the assistance of our Department of Dentistry, as personnel there are quite familiar and experienced with the procedure. Once an impression had been created, each patient presented for initial setup on the Gamma Knife Perfexion. The dental tray was attached to the Extend frame, and a comfortable fit between the patient, frame, and Perfexion Patient Positioning System was created. Any devices used to assist patient comfort (wedge pillows under the knees, for example) were noted by the treatment team. The Extend frame was then tightened using a torque wrench. Finally, air was evacuated from the vac-loc-type head cushion behind the patient's head. 


\section{Positioning uncertainty of the Gamma Knife Extend system}

TABLE 1: Demographics, treatment plan characteristics, and mean positioning results across all fractions for the 10 patients included in the study

\begin{tabular}{|c|c|c|c|c|c|c|c|c|}
\hline $\begin{array}{l}\text { Case } \\
\text { No. }\end{array}$ & $\begin{array}{l}\text { Sex, Age } \\
\left(\mathrm{yrs}^{\star}\right)\end{array}$ & Diagnosis & $\begin{array}{l}\text { Prescribed Isodose } \\
\qquad \mathrm{Vol}\left(\mathrm{cm}^{3}\right)\end{array}$ & $\begin{array}{l}\text { Total Prescribed } \\
\text { Dose (Gy) } \dagger\end{array}$ & $\begin{array}{c}\text { No. of } \\
\text { Fractions }\end{array}$ & $\begin{array}{l}\text { Beam-On } \\
\text { Time (min) } \ddagger\end{array}$ & $\begin{array}{l}\text { Setup Difference } \\
(\mathrm{mm}) \S\end{array}$ & $\begin{array}{l}\text { Intrafraction Difference } \\
(\mathrm{mm}) \S\end{array}$ \\
\hline 1 & $F, 43$ & meningioma & 2.99 & 20 & 4 & 21.0 & $0.83 \pm 0.07$ & $0.84 \pm 0.42$ \\
\hline 3 & $F, 49$ & meningioma & 5.52 & 20 & 4 & 34.3 & $0.46 \pm 0.24$ & $0.39 \pm 0.04$ \\
\hline 4 & M, 47 & CN V schwannoma & 10.46 & 20 & 4 & 46.8 & $0.55 \pm 0.17$ & $0.68 \pm 0.26$ \\
\hline 5 & $F, 68$ & meningioma & 15.71 & 18 & 3 & 46.8 & $0.61 \pm 0.13$ & $0.32 \pm 0.15$ \\
\hline 7 & $\mathrm{M}, 57$ & meningioma & 11.98 & 18 & 4 & 36.8 & $0.90 \pm 0.19$ & $0.49 \pm 0.20$ \\
\hline 8 & $F, 69$ & meningioma & 22.69 & 18 & 3 & 14.2 & $0.86 \pm 0.12$ & $0.33 \pm 0.19$ \\
\hline 9 & $\mathrm{M}, 73$ & meningioma & 2.17 & 20 & 4 & 13.9 & $0.56 \pm 0.28$ & $0.21 \pm 0.06$ \\
\hline 10 & $M, 54$ & meningioma & 1.3 & 18 & 3 & 17.2 & $0.66 \pm 0.06$ & $0.52 \pm 0.18$ \\
\hline
\end{tabular}

* Age at time of first fraction. Abbreviation: $\mathrm{CN}=$ cranial nerve.

$\dagger$ Dose prescribed to $50 \%$ isodose line.

$\ddagger$ Beam-on time at the date of the first fraction. Beam time increases slightly over treatment due to the ${ }^{60} \mathrm{Co}$ decay.

$\S$ Values are expressed as means \pm SDs.

Once the patient had been placed in position, notations of anatomically significant landmarks, such as the tragus, height of the patient's chin, and so forth, were made to assist in future repositioning. Using the digital measurement probes, a provisional set of measurements was taken for comparison against later measurements.

Following the initial setup at the Gamma Knife, each patient was transferred to the MR imaging suite, for volumetric imaging deemed appropriate for the particular indication, and then to the CT suite for stereotactic imaging. Magnetic resonance imaging was performed without the Extend frame. Computed tomography scanning required the frame and a stereotactic localizer box, and the patient's position at the time of CT imaging served as the reference position for the patient for all subsequent treatment sessions. To establish this reference position, the patient was placed on the CT table with the Extend frame in place. Every effort was made to reproduce the patient's position as it was at the initial Gamma Knife setup.

\section{Reference Position Measurements}

With the patient in the intended treatment position, the RCT and measurement probes were used to record the position of the patient's head within the Extend frame. The vacuum surveillance system on the PCU was activated. Reference measurements were obtained using the digital probes, which were placed through measurement holes in the RCT. At least three holes were selected on each plate (anterior, superior, left, and right plates) of the $\mathrm{RCT}$, at locations where the probe tips could be perpendicular to the patient's skull and avoid areas of loose skin and fat (Fig. 2). The hole identification code, measurement result, and probe type (long or short) were recorded.

Following measurement of the reference position, stereotactic CT images were acquired using the stereotactic localization box. Following imaging, position measurements were repeated to verify that the patient remained immobile during imaging. A 1-mm radial error was used as a threshold for repositioning and reimaging. No patient in this study exceeded this threshold.

Once imaging had been completed, the images were imported into the Gamma Knife treatment planning system (GammaPlan version 9.0, Elekta AB). The MR and CT imaging studies were coregistered using a rigid coregistration $^{23}$ routine provided with the software. Treatment planning then proceeded, as described previously for $\mathrm{GKS},{ }^{7}$ with the exception that a radiation dose composed of multiple fractions was prescribed.

\section{Residual Setup Difference and Intrafraction Difference}

Immediately before delivery of each fraction of radiation, the patient was placed on the Gamma Knife Pa-

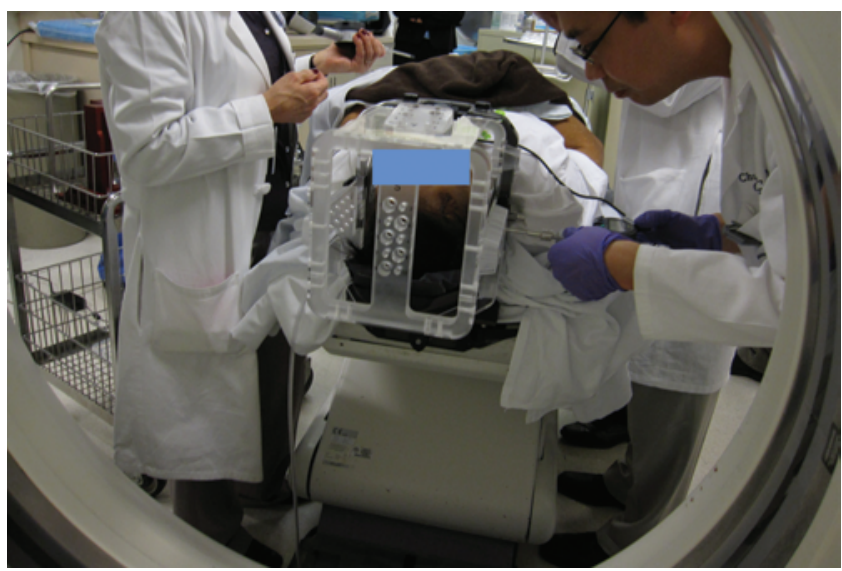

FIG. 2. Photograph showing measurement of the patient's position within the Extend Frame using digital measurement probes and the RCT. Reference measurements are obtained at the time of CT simulation. Repositioning measurements are obtained at the Gamma Knife before each fraction and compared with reference measurements to estimate setup uncertainty. Measurements are again obtained at the conclusion of each fraction and compared with measurements obtained before the fraction to estimate intrafraction uncertainty. 
tient Positioning System with the Extend frame, vacuum cushion, and PCU. With the vacuum surveillance system active, repositioning measurements were obtained using digital probes and measurement holes selected on the RCT at the time of CT simulation. The difference between repositioning measurements and reference measurements were computed and averaged for each plate of the RCT. A radial deviation from the reference position was calculated as:

$$
\Delta_{\text {radial }}=\sqrt{\left(\Delta_{\text {anterior }}\right)^{2}+\left(\Delta_{\text {superior }}\right)^{2}+\left(\frac{\Delta_{\text {left }}-\Delta_{\text {right }}}{2}\right)^{2}}
$$

where $\Delta_{\text {anterior }}, \Delta_{\text {superior }}, \Delta_{\text {left }}$, and $\Delta_{\text {right }}$ are the mean values for each plate of the RCT.

A clinical threshold of $1.0 \mathrm{~mm}$ radial deviation was maintained for repositioning. If this threshold was exceeded, patient positioning was repeated, and new repositioning measurements were acquired until the patient's position was within the threshold. The final radial difference between the patient's prefraction position and the reference position was recorded as the "residual setup difference."

Delivery of the treatment fraction proceeded according to the treatment plan. During radiation delivery, if the vacuum level monitored by the PCU decreased by more than a threshold (10\%), the system automatically paused treatment delivery. In this instance the patient was repositioned within the Extend frame, repositioning measurements were repeated, and treatment was resumed.

At the completion of each treatment fraction, repositioning measurements were again acquired using the RCT and digital probe system. The radial difference between the new measurements and the measurements of patient position made before the treatment fraction was recorded as the "intrafraction difference." Because we are interested in the uncertainty caused by intrafraction motion that is not detected by the vacuum monitoring system, we excluded from the analysis any fraction in which the vacuum monitoring system paused treatment during delivery and the patient was repositioned.

\section{Systematic and Random Uncertainty Components}

For both the setup and intrafraction positional differences, the distribution of systematic $(\Sigma)$ and random $(\sigma)$ uncertainties were calculated following the method of Stroom and Heijmen. ${ }^{18}$ If, for each treatment fraction, the mean setup displacement for patient $\mathrm{x}$ is $\mathrm{m}_{\mathrm{x}}$ with a standard deviation $\mathrm{sd}_{\mathrm{x}}$, then

Distribution of systematic uncertainty $(\Sigma)=$ standard deviation of all means $\left(\mathrm{m}_{\mathrm{x}}\right)$

Distribution of random uncertainty $(\sigma)=$ root mean square (RMS) of all standard deviations $\left(\mathrm{sd}_{\mathrm{x}}\right)$

(Fig. 3). The distributions of random and systematic uncertainty were computed for each plate of the RCT, as well as for the overall radial differences. These are reported along with the group mean, group standard deviation for all patients over all fractions, and the RMS of the differences for each plate.

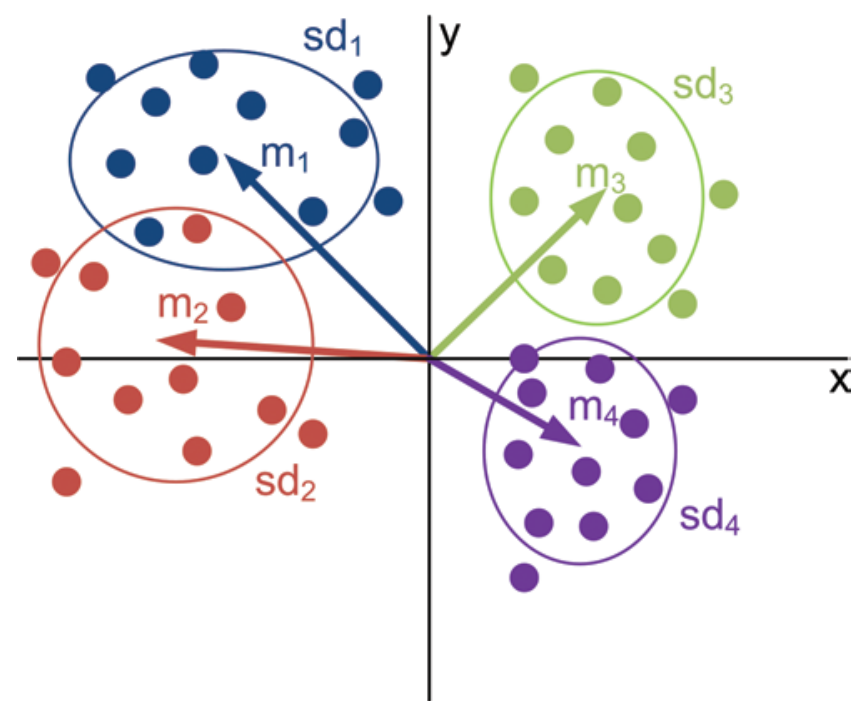

FIG. 3. Schematic drawing illustrating the definitions of systematic and random uncertainty. Individual fraction measurements are represented by the dots in the diagram. Each patient has a distribution of dots, with a mean $m_{x}$ and a standard deviation $\mathrm{sd}_{\mathrm{x}}$. Style of representation patterned on Figure 2 from Stroom JC, Heijmen BJ: Radiother Oncol 64:75-83, 2002.

\section{Results}

All 10 patients completed all treatment fractions as planned by the treatment team. In all cases, the patient was able to be repositioned to within the 1 -mm radial displacement action level. Thus, no patient required a new CT scan or revision of the treatment plan.

During delivery of a single fraction to 1 patient, the vacuum interlock automatically paused treatment, indicating possible patient motion during treatment delivery. This patient was repositioned to within the $1-\mathrm{mm}$ action threshold, and treatment was resumed uneventfully. For the reasons described earlier, this fractional treatment was excluded from the intrafraction uncertainty results.

\section{Setup Uncertainty}

Table 1 summarizes the mean setup and intrafraction positioning differences for each patient. Figure 4 illustrates the setup uncertainty results for each plate of the RCT across all patients and all 36 fractions, histograms of the mean measurements obtained for each plate, and the overall radial setup uncertainty. The mean radial setup difference was $0.64 \mathrm{~mm}$ (SD $0.24 \mathrm{~mm})$. The distribution of the systematic uncertainty component $(\Sigma)$ was $0.17 \mathrm{~mm}$, and the distribution of the random component $(\sigma)$ was 0.16 $\mathrm{mm}$. On a per-plate basis, the mean positional differences were -0.05 for the anterior plate, $-0.11 \mathrm{~mm}$ for the superior plate, $0.09 \mathrm{~mm}$ for the left plate, and $-0.01 \mathrm{~mm}$ for the right plate. The RMSs of the differences per plate were 0.20 for the anterior plate, $0.28 \mathrm{~mm}$ for the superior plate, $0.41 \mathrm{~mm}$ for the left plate, and $0.35 \mathrm{~mm}$ for the right plate.

\section{Intrafraction Uncertainty}

Figure 5 shows the corresponding information for the intrafraction uncertainty on each plate of the RCT across 


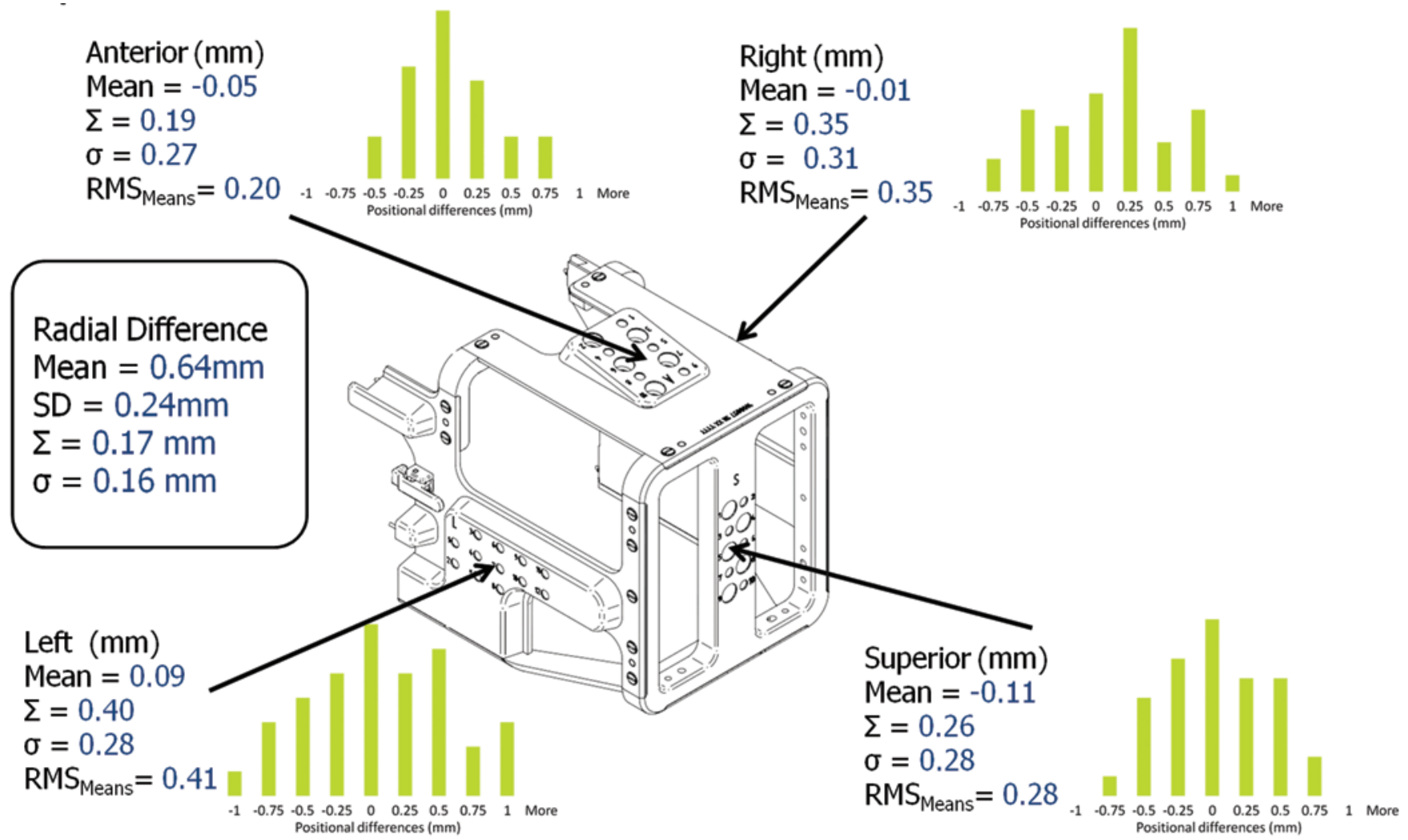

FIG. 4. Illustration and bar graphs showing the means and standard deviations of the positional differences between prefraction measurements and the reference measurements, as well as the associated uncertainty distributions for each plate of the RCT. The RCT illustration is reproduced courtesy of Elekta AB.

all treatments and 35 fractions. The mean radial positional difference was $0.47 \mathrm{~mm}$ (SD $0.30 \mathrm{~mm}$ ), with $\Sigma=0.18 \mathrm{~mm}$ and $\sigma=0.22 \mathrm{~mm}$. On a per-plate basis, the mean differences were -0.27 for the anterior plate, $-0.06 \mathrm{~mm}$ for the superior plate, $0.09 \mathrm{~mm}$ for the left plate, and $0.13 \mathrm{~mm}$ for the right plate. The RMSs of the differences per plate were 0.34 for the anterior plate, $0.24 \mathrm{~mm}$ for the superior plate, $0.22 \mathrm{~mm}$ for the left plate, and $0.24 \mathrm{~mm}$ for the right plate.

\section{Discussion}

The results presented in our work are similar in magnitude to results reported for other dental fixationbased immobilization devices (Table 2). Sweeney and colleagues ${ }^{19}$ evaluated the repositioning performance of a vacuum-assisted dental fixation device. Surface landmarks measured using an optical neuronavigation system served as a basis for positioning accuracy. The mean baseline difference between planned and achieved landmark positions (system error, operator error, and patient movement prior to repositioning) was $0.57 \mathrm{~mm}$, and the mean repositioning difference was $1.02 \mathrm{~mm}$. It should be noted that the repositioning difference in that case was not a residual difference, but rather the difference obtained each time the patient was placed within the frame. For quality assurance purposes, Kunieda et al. ${ }^{6}$ evaluated a similar device that is commercially available (HeadFix, Elekta $\mathrm{AB}$ ), matching anatomical landmarks on CT scans obtained throughout the course of treatment to correspond- ing landmarks on treatment-planning $\mathrm{CT}$ scans. For the various landmarks used by those researchers, the mean radial positional displacements ranged from 0.93 to 1.09 $\mathrm{mm}$ (SD $0.52-0.88 \mathrm{~mm}$ ), with rotations of $0.49 \pm 0.36^{\circ}$.

Meeks and coauthors ${ }^{11}$ presented the results of a performance study of a frameless system consisting of a bite plate containing fiducial markers made of both aluminum (visible on CT) and infrared LEDs (visible using an infrared tracking system) with a known, fixed positional correspondence. At the time of patient simulation, the simulation CT scanner provided actual aluminum and calculated infrared LED fiducial positions. At patient setup and during treatment delivery, the actual infrared LED-defined positions were compared against desired positions determined at the time of simulation. The authors report positioning differences of $0.65 \mathrm{~mm} \pm 0.17$ $\mathrm{mm}$ (SD) when a test phantom was used and $1.1 \pm 0.3 \mathrm{~mm}$ (SD) when patients were examined in a clinical setting.

The same group ${ }^{14}$ reported on a commercially available version of the system (RadioCameras, Zmed, and Varian), which replaces the infrared LEDs with passive reflective markers and a camera equipped with infrared illuminators. In that study of 15 patients, the authors found the mean total predicted localization difference from simulation to initial setup to be $0.82 \pm 0.41 \mathrm{~mm}$ (SD), including the biteblock reseating error and the image registration error of the reflective fiducial markers. This difference was then reduced to a mean of $0.16 \pm 0.04$ $\mathrm{mm}$ (SD) using adjustments on the device. 


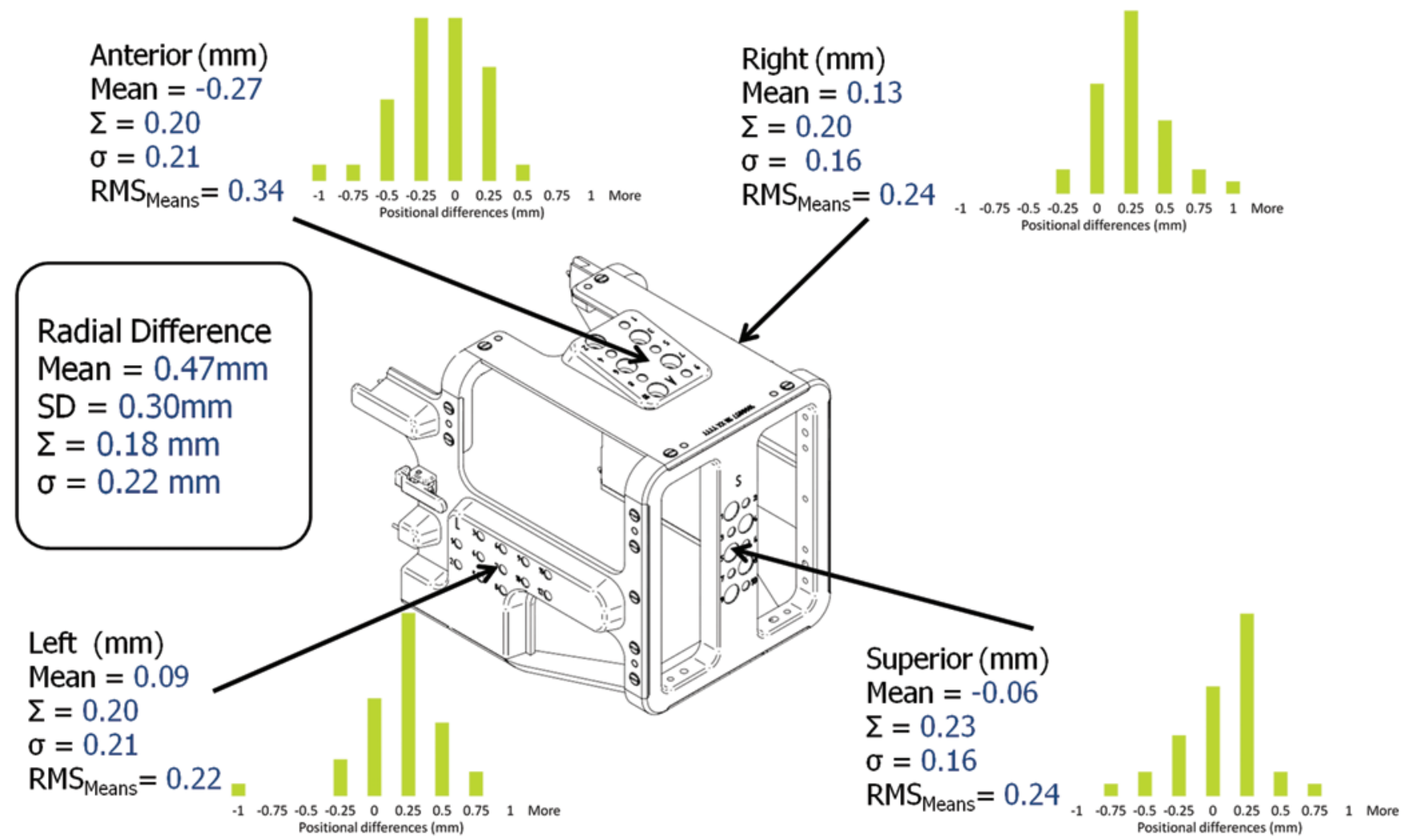

FıG. 5. Illustration and bar graphs showing the means and standard deviations of the positional differences between prefraction and postfraction measurements, as well as the associated uncertainty distributions for each plate of the RCT. The RCT illustration is reproduced courtesy of Elekta AB.

Baumert, et al. ${ }^{1}$ investigated a combination thermoplastic mask-dental fixation system and reported a mean setup error of $2.2 \pm 1.1 \mathrm{~mm}(\mathrm{SD})$ when they compared the planning CT scan with a "check" CT scan obtained after patient setup in the treatment position.

Ruschin et al. ${ }^{13}$ reported the results of testing performed on a preclinical version of the Extend system, using a linear accelerator with onboard imaging. In healthy volunteers, there was a mean residual setup difference of $0.6 \pm 0.3 \mathrm{~mm}(\mathrm{SD})$, based solely on measurements obtained by the RCT and digital probes. In patients, there was a mean displacement of $1.3 \mathrm{~mm}(\Sigma=0.9 \mathrm{~mm}, \sigma=0.4$ $\mathrm{mm}$ ) and an intrafraction displacement of $0.4 \pm 0.3 \mathrm{~mm}$.

To put these results into perspective, the overall un- certainty in setup and immobilization of traditional frame systems has been reported to be between 1.0 and $2.0 \mathrm{~mm}$, depending on the technique used to evaluate the uncertainty and the sources of uncertainty included in the analysis., ${ }^{92,16,25}$ Relocatable frames, such as the Gill-ThomasCosman frames, reportedly have uncertainties of $2.0 \mathrm{~mm}^{2}$ and thermoplastic masks in the range of $1.6-3.2 \mathrm{~mm} .{ }^{2,3}$

In our series we found an overall mean radial setup displacement of $0.64 \mathrm{~mm}$ and an overall mean intrafraction displacement of $0.47 \mathrm{~mm}$. This indicates that the Extend system performs as expected for a device of its class. With careful patient selection and care in setup, the Extend immobilization system is adequate for use in a multisession radiosurgical setting. This is not to say that the

TABLE 2: A summary of reported setup uncertainty results on immobilization devices similar to the Extend system

\begin{tabular}{llcl}
\hline \multicolumn{1}{c}{ Authors \& Year } & Device & Mean Displacement (mm) & Comparison Technique $^{*}$ \\
\hline Sweeney et al., 1998 & dental fixation + vacuum assist & 1.02 & surface landmarks (neuronavigation probe) \\
Meeks et al., 2000 & $\begin{array}{c}\text { infrared LED tracked bite plate + CT } \\
\text { fiducial markers }\end{array}$ & $\begin{array}{c}0.65 \pm 0.17 \text { phantom } \\
1.1 \pm 0.3 \text { patient }\end{array}$ & infrared-LED positions vs CT fiducial positions \\
Ryken et al., 2001 & $\begin{array}{c}\text { RadioCameras (infrared-tracked reflector } \\
\text { bite plate) }\end{array}$ & $0.16 \pm 0.04$ & optically determined fiducial positions vs CT positions \\
Baumert et al., 2005 & thermoplastic mask + dental fixation & $2.2 \pm 1.1$ & simulation CT vs quality assurance CT \\
Kuneida et al., 2009 & HeadFix (dental fixation + vacuum assist) & $0.93-1.09 \pm 0.52-0.88$ & anatomical landmarks (treatment CT vs simulation CT) \\
Ruschin et al., 2010 & prototype Extend system & 1.3 & RCT measurements vs cone-based CT \\
\hline
\end{tabular}

\footnotetext{
${ }^{*}$ Value expressed in means \pm SDs if reported.
} 


\section{Positioning uncertainty of the Gamma Knife Extend system}

system is suitable for all patients, however. In particular, patients who exhibit an altered neurological state, lower cranial nerve dysfunction, respiratory difficulties, poor or no dentition, oropharyngeal problems, or extensive skull or scalp irregularities may not achieve adequate setup when the Extend system is used.

\section{Margins and Multisession SRS}

A number of margin formulas ${ }^{4,5,10,18,22}$ appear in the literature in which the researcher attempts to use the distributions of random and systematic uncertainty to calculate optimal treatment margins for external beam, intensity-modulated, and stereotactic body radiation treatments. Perhaps the best known is the margin formula by van Herk et al., ${ }^{21}$ who propose the formula

"margin $=2.5 \Sigma+0.7 \sigma "$

to calculate a margin that would result in $90 \%$ of patients receiving the full planned dose (usually described as 95\% of the prescription dose) to the clinical target volume. If we apply the van Herk formula to our setup and intrafraction results, adding each $\Sigma$ and $\sigma$ for the setup and intrafraction uncertainty in quadrature, ${ }^{20}$ we will arrive at a required margin of $0.81 \mathrm{~mm}$. However, GKS violates many of the underlying assumptions of the van Herk formula, such as an infinite number of fractions, the prescription isodose line customarily used, and the width of the beam penumbra. ${ }^{4}$ More work is required to adapt the van Herk formula to the situation of GKS usng the Extend system.

\section{Limitations of the Current Study}

There are several aspects of this study that may limit the general applicability of our conclusions.

Effect of Rotations. The RCT template has 4 plates that allow measurements to be taken parallel to the primary orthogonal planes (anterior-posterior, left-right, and superior-inferior). However, the system has no way of explicitly accounting for rotations. Patients with skulls that are highly spherical could be positioned well, based on the probe or RCT measurements, and still be out of position due to these rotations. In our study, the effect of rotations is partially accounted for by using a minimum of three measurement holes per plate of the RCT, distributed over the surface of the patient's head. Because, a patient's skull is rarely spherical, the distribution of measurements makes it likely that rotations would implicitly manifest in the resulting repositioning measurements. It may also be the case that for intracranial cases, explicitly accounting for rotations may not be critical. Ma and colleagues ${ }^{8}$ compared patient setup differences between 3-dimensional matching (translational correction only) and 6-dimensional matching (translational and rotational correction) using a 6-degree of freedom treatment table. Cone-beam CT imaging of the actual patient position yielded no statistical difference in rotation between the two correction methods.

Assumption of Rigidity. The RCT-digital probe system assumes that measurements of cranial position within the frame can be an accurate surrogate for the position of the radiosurgical target and surrounding critical structures. This assumption has been called into question, notably by Ruschin et al., ${ }^{13}$ who found that when comparing linear probe measurements of the skull to imaging of the head position using the onboard cone-beam CT of a linear accelerator, the brain could sometimes be shifted out of position in a direction opposite to that described by the measurements. Since the Gamma Knife currently has no onboard imaging guidance, we are forced to accept the assumption that the brain and skull are a single rigid system.

Patient Selection Bias. This study was performed in a relatively small number of patients who were selected specifically to receive Extend treatments. At our center, patients are only referred for Extend-based treatments if they have a high performance status, are reasonably compliant, and are therefore likely to accept the physical manipulation required to create a dental mold and be positioned for treatment. In all 10 cases the radiosurgical targets were benign tumors and the patients were otherwise considered healthy. This patient cohort is therefore unlikely to be representative of an overall cohort of patients treated with radiosurgery, using either the Gamma Knife or another radiosurgical device.

Vacuum Detection System. The Extend system relies on the level of suction between the patient's dental mold and upper palate as a surrogate to determine patient motion. If the suction level drops, the system assumes the patient has moved out of position and treatment is paused. As described earlier, one such event occurred during the study; however, there was no way to know whether the vacuum was triggered by a momentary excursion of the patient from the intended position, a sustained excursion, or some other factor that could cause a loss of suction. To our knowledge, the reliability and sensitivity of the vacuum system to patient motion has not been published in the literature and is a topic of ongoing investigation at our center.

Other Sources of Uncertainty. The reader should be reminded that patient position is only one of many sources of uncertainty in the overall uncertainty budget of a GKS procedure. Uncertainty in imaging, contouring, dosimetry, and treatment delivery all play a role in overall uncertainty. ${ }^{16}$ These additional sources of uncertainty are not taken into account in this study, but they should be included in any subsequent margin calculation.

\section{Conclusions}

With care in patient selection and initial setup, the Gamma Knife Extend system provides a reasonable assurance of setup accuracy and immobilization for multisession radiosurgery. While the system does not provide any direct measure of rotation, the use of multiple measurements per RCT plate may implicitly take rotations into account. Further work is needed to characterize the sensitivity of the vacuum monitoring system to detect patient motion. Care in the initial positioning of the patient and attention to patient comfort helps minimize the increased treatment overhead required by the system. 


\section{Disclosure}

The authors report no conflict of interest concerning the materials or methods used in this study or the findings specified in this paper.

Author contributions to the study and manuscript preparation include the following. Conception and design: Schlesinger, Yen. Acquisition of data: Schlesinger, Xu, Taylor, Yen, Sheehan. Analysis and interpretation of data: Schlesinger, Xu, Taylor, Sheehan. Drafting the article: Schlesinger. Critically revising the article: Schlesinger, $\mathrm{Xu}$, Taylor, Sheehan. Reviewed submitted version of manuscript: Sheehan. Approved the final version of the manuscript on behalf of all authors: Schlesinger. Statistical analysis: Schlesinger. Study supervision: Sheehan.

\section{Acknowledgments}

The authors thank Greg Patterson and Jean-Claude Matte for their assistance with documenting clinical Extend system treatments.

\section{References}

1. Baumert BG, Egli P, Studer S, Dehing C, Davis JB: Repositioning accuracy of fractionated stereotactic irradiation: assessment of isocentre alignment for different dental fixations by using sequential CT scanning. Radiother Oncol 74:61-66, 2005

2. Bednarz G, Machtay M, Werner-Wasik M, Downes B, Bogner J, Hyslop T, et al: Report on a randomized trial comparing two forms of immobilization of the head for fractionated stereotactic radiotherapy. Med Phys 36:12-17, 2009

3. Fuss M, Salter BJ, Cheek D, Sadeghi A, Hevezi JM, Herman TS: Repositioning accuracy of a commercially available thermoplastic mask system. Radiother Oncol 71:339-345, 2004

4. Gordon JJ, Siebers JV: Convolution method and CTV-to-PTV margins for finite fractions and small systematic errors. Phys Med Biol 52:1967-1990, 2007

5. International Commission for Radiation Units and Measurements: ICRU Report 62. Prescribing, recording, and reporting photon beam therapy (Supplement to ICRU Report 50). Bethesda, MD: ICRU, 1999

6. Kunieda E, Oku Y, Fukada J, Kawaguchi O, Shiba H, Takeda A, et al: The reproducibility of a HeadFix relocatable fixation system: analysis using the stereotactic coordinates of bilateral incus and the top of the crista galli obtained from a serial CT scan. Phys Med Biol 54:N197-N204, 2009

7. Lindquist C, Paddick I: The Leksell Gamma Knife Perfexion and comparisons with its predecessors. Neurosurgery 61 (3 Suppl):130-141, 2007

8. Ma J, Chang Z, Wang Z, Jackie Wu Q, Kirkpatrick JP, Yin FF: ExacTrac X-ray 6 degree-of-freedom image-guidance for intracranial non-invasive stereotactic radiotherapy: comparison with kilo-voltage cone-beam CT. Radiother Oncol 93: 602-608, 2009

9. Maciunas RJ, Galloway RL Jr, Latimer J, Cobb C, Zaccharias E, Moore A, et al: An independent application accuracy evaluation of stereotactic frame systems. Stereotact Funct Neurosurg 58:103-107, 1992

10. McKenzie AL, van Herk M, Mijnheer B: The width of margins in radiotherapy treatment plans. Phys Med Biol 45:33313342,2000

11. Meeks SL, Bova FJ, Wagner TH, Buatti JM, Friedman WA, Foote KD: Image localization for frameless stereotactic radiotherapy. Int J Radiat Oncol Biol Phys 46:1291-1299, 2000

12. Rosenzweig DP, Schell MC, Numaguchi Y: Quality assurance in linac-based stereotactic radiosurgery and radiotherapy. Med Dosim 23:147-151, 1998
13. Ruschin M, Nayebi N, Carlsson P, Brown K, Tamerou M, Li $\mathrm{W}$, et al: Performance of a novel repositioning head frame for gamma knife perfexion and image-guided linac-based intracranial stereotactic radiotherapy. Int J Radiati Oncol Biol Phy 78:306-313, 2010

14. Ryken TC, Meeks SL, Pennington EC, Hitchon P, Traynelis $\mathrm{V}$, Mayr NA, et al: Initial clinical experience with frameless stereotactic radiosurgery: analysis of accuracy and feasibility. Int J Radiat Oncol Biol Phys 51:1152-1158, 2001

15. Sayer FT, Sherman JH, Yen CP, Schlesinger DJ, Kersh R, Sheehan JP: Initial experience with the eXtend System: a relocatable frame system for multiple-session gamma knife radiosurgery. World Neurosurg 75:665-672, 2011

16. Schell M, Bova FJ, Larson DA, Leavitt DD, Lutz WR, Podgorask EB, et al: AAPM Report No. 54, Stereotactic Radiosurgery. Woodbury, NY: American Institute of Physics, 1995 (http://www.aapm.org/pubs/reports/rpt_54.pdf) [Accessed June 25, 2012]

17. Solberg TD, Medin PM, Mullins J, Li S: Quality assurance of immobilization and target localization systems for frameless stereotactic cranial and extracranial hypofractionated radiotherapy. Int J Radiat Oncol Biol Phys 71 (1 Suppl):S131S135, 2008

18. Stroom JC, Heijmen BJ: Geometrical uncertainties, radiotherapy planning margins, and the ICRU-62 report. Radiother Oncol 64:75-83, 2002

19. Sweeney R, Bale R, Vogele M, Nevinny-Stickel M, Bluhm A, Auer T, et al: Repositioning accuracy: comparison of a noninvasive head holder with thermoplastic mask for fractionated radiotherapy and a case report. Int J Radiat Oncol Biol Phys 41:475-483, 1998

20. Taylor BN, Kuyatt CE: NIST Technical Note 1297: Guidelines for Evaluating and Expressing the Uncertainty of NIST Measurement Results. Gaithersburg, MD: National Institute of Standards and Technology, 1994 (http://physics. nist.gov/Pubs/guidelines/TN1297/tn1297s.pdf) [Accessed June 25, 2012]

21. van Herk M, Remeijer P, Lebesque JV: Inclusion of geometric uncertainties in treatment plan evaluation. Int J Radiat Oncol Biol Phys 52:1407-1422, 2002

22. van Herk M, Witte M, van der Geer J, Schneider C, Lebesque $\mathrm{JV}$ : Biologic and physical fractionation effects of random geometric errors. Int J Radiat Oncol Biol Phys 57:1460-1471, 2003

23. Wells WM III, Viola P, Atsumi H, Nakajima S, Kikinis R: Multi-modal volume registration by maximization of mutual information. Med Image Anal 1:35-51, 1996

24. Wu A: Physics and dosimetry of the gamma knife. Neurosurg Clin N Am 3:35-50, 1992

25. Yeung D, Palta J, Fontanesi J, Kun L: Systematic analysis of errors in target localization and treatment delivery in stereotactic radiosurgery (SRS). Int J Radiat Oncol Biol Phys 28:493498, 1994

Manuscript submitted May 14, 2012.

Accepted June 22, 2012.

Portions of this work were presented at the 16th International Leksell Gamma Knife Society Meeting, Sydney, Australia, March 26, 2012.

Please include this information when citing this paper: DOI: 10.3171/2012.6.GKS12989.

Address correspondence to: David Schlesinger, Ph.D., University of Virginia Gamma Knife Center, 1215 Lee Street, Suite G512, Box 800742, Charlottesville, Virginia 22908-0742. email: djs9c@ virginia.edu. 